\begin{tabular}{|c|l|}
\hline Title & Parallel evolution of novelties: extremely long intromittent organs in the leaf beetle subfamily Criocerinae \\
\hline Author(s) & Matsumura, Yoko; Machida, Ry uichiro; Wipfler, Benjamin; Beutel, Rolf G.; Y oshizawa, Kazunori \\
\hline Citation & $\begin{array}{l}\text { Evolution \& Development, 15(4), 305-315 } \\
\text { https://doi.org/10.1111/ede.12036 }\end{array}$ \\
\hline Issue Date & 2013-07 \\
\hline Doc URL & http://hdl.handle.net/2115/56847 \\
\hline Type & article (author version) \\
\hline File Information & 2013evodevo.pdf \\
\hline
\end{tabular}

Instructions for use 


\section{Parallel evolution of novelties: extremely long intromittent organs in the leaf beetle subfamily Criocerinae}

Running head: morphogenesis of aedeagus in beetles

Yoko Matsumura ${ }^{1,2}$, Ryuichiro Machida ${ }^{3}$, Benjamin Wipfler ${ }^{2}$, Rolf G. Beutel ${ }^{2}$, Kazunori Yoshizawa $^{1}$

(affiliation of the authors. 1: Laboratory of Systematic Entomology, Department of Ecology and Systematics, Graduate School of Agriculture, Hokkaido University, Sapporo, Japan. 2: Entomology Group, Institut für Spezielle Zoologie und Evolutionsbiologie mit Phyletischem Museum, FSU Jena, Germany. 3: Sugadaira Montane Research Center, University of Tsukuba, Nagano, Japan)

Corresponding author' information: Y. Matsumura: Entomology Group, Institut für Spezielle Zoologie und Evolutionsbiologie mit Phyletischem Museum, FSU Jena, Germany; telephone 03641949184; FAX 03641949142; yoko.matumura.hamupeni@gmail.com)

The number of words: 6622 . 


\begin{abstract}
Extreme elongation of a part of the intromittent organ, the flagellum, has occurred several times in Criocerinae (Chrysomelidae). These leaf beetles have acquired a specialized pocket to store the flagellum in the abdominal cavity, at the same time allowing a quick control of movements of this structure during copulation. We investigated the morphogenesis of the intromittent organs of species with and without a flagellum to discuss the evolutionary background of parallel evolution of novel structures. We found that the specialized pocket is formed by the invagination of an epidermal layer and a resultant rotation of the primary gonopore. Invagination itself is a well-known phenomenon in morphogenetic processes, which leads us to hypothesize that the novelty is formed by co-opting a previously acquired genetic system. A large open-space is present within the intromittent organ during the entire morphogenesis in species without a flagellum, and the invagination in the species with a flagellum grows in the corresponding area. This means that there are no physical impediments for the growth of a large pocket. In addition the sites of muscular attachments in the species with a flagellum are also different from those without it. The differentiation of muscles is completed immediately before adult emergence, which means the muscles are adjustable during the entire morphogenesis in this group. Simple modifications probably based on a co-option of previously acquired genetic systems, the potential space for adding a new element, and an adjustable factor in morphogenesis of the intromittent organ facilitate the parallel evolution of the extreme elongation.
\end{abstract}




\section{Introduction}

The origin of novelties is still an insufficiently understood phenomenon in evolutionary biology (Mayr 1960; Müller and Wagner 1991, 2003; Moczek 2008; Brakefield 2011; Hendrickson and Rainey 2012). Recent investigations of evolutionary developmental biologists suggest that genetic accommodation, expression of developmental capacitance, and modifications and/or co-option of previously acquired regulatory systems of developments are involved, in addition to newly acquired developmental pathways (e.g. Mayr 1960; Kirschner and Gerhart 2005; Brakefield 2006, 2011; Moczek 2007, 2008; Shubin et al. 2009; Müller 2010; Scotland 2010; Blount et al. 2012). As the evolution of organisms results from the modification of descendants, these mechanisms are reasonable (Brakefield 2011). However, the morphogenesis of structures of an organism must progress harmoniously with neighboring organs and tissues. This implies that large scale alterations of the morphogenesis of certain elements can be detrimental for entire organs or organisms, and opens the question of how novel characters can be acquired without a reduction of the overall fitness (e.g. Mayr 1960; Roth and Wake 1989; Müller and Wagner 2003). This applies to single events but even to a much higher degree to similar innovations evolving several times independently.

Detecting developmental changes causing novelties is essential for uncovering the mechanisms of emerging novel forms (Müller and Wagner 1991). The evolutionary processes linked with structural novelties were discussed in several recent studies from a developmental perspective (e.g. Brylski and Hall 1988; Prum 1999; Page 2000; Kaji and Tsukagoshi 2008, 2010; Nagashima et al. 2009; Kaji 2010, 2012; Tajiri et al. 2010; Kaji et al. 2011, 2012; Atllah et al. 2012; Dugon et al. 2012). It was clearly shown that the origin of novelties can result from much simple modifications of adjustable elements. However, most of these previous studies were focused on single innovations. In the present study we aim at a better understanding of morphogenetic changes resulting in the parallel evolution of similar novelties. As an exemplar character system we use extremely elongated male intromittent organs, which have independently evolved in several groups of the leaf beetle subfamily Criocerinae.

Extreme elongation of the intromittent organ itself or parts of the male intromittent organ occurs in many groups of animals as for instance in ducks, limpets, barnacles, ostracods, and many arthropods (summarized in Neufeld and Palmer 2008; Matsumura and Yoshizawa 2012). In insects this phenomenon is known in several lineages. The length of the elongated part of the intromittent organ is greatly variable among species, but unlike in mammals, it is constant within 
individuals during their entire adult life span. It is usually stored within the abdomen in pterygote insects (Snodgrass 1935), even in species with elongated elements often even exceeding the total body length. Using criocerine leaf beetles Matsumura and Yoshizawa $(2010,2012)$ revealed that species with an extremely elongated part (= flagellum: a terminal elongation of the ejaculatory duct; Lindroth 1957, see Box 1 for a terminological problem) of the aedeagus (the intromittent organ of insects, Fig. 1) have evolved a highly specialized configuration to control the movements of the thin and tubular flagellum during copulation and for its storage it in the very limited space in their abdomen (Fig. 2, see also Fig. 1 for terminology). It was proposed that the derived conditions have evolved at least three times in the subfamily (Fig. 2A), and the assumed pattern of transformation from a plesiomorphic state was similar in the three clades (= rotation of sclerites along the craniocaudal axis; Matsumura and Yoshizawa 2012).

In the present study we discuss how the novelty was acquired several times independently. The morphogenesis of the intromittent organ was investigated in detail focusing on one of the clades (Fig. 2E, the subgenus Lema) and the findings are compared with conditions in the other relevant taxa. The processes underlying the parallel evolution of highly modified intromittent organs and the possibility that most involved novel features can be explained by a co-option of previously acquired genetic regulatory systems in this subfamily are discussed.

[Box1. The term "flagellum" has been variously used to describe thin and elongated structures in biology, as for instance the tail of spermatozoa, locomotor appendages of bacteria and protozoa, the multisegmented distal part of insect antennae, a part of intromittent organs in insects and so on. This term can have very different meanings depending on the context. However, as "flagellum" is frequently used to describe the terminal elongation of the ejaculatory duct (see Fig. 1), we decided to maintain it here with a clear definition, in order to minimize nomenclatural confusion. ]

\section{Materials and Methods}

\section{(a) Beetle species examined and rearing}

We used the following four species: Lema (Lema) coronata, L. (L.) diversa, Lema (Microlema) decempunctata, Oulema oryzae. The first two species have the tubular flagellum and a specialized pocket for storing it (Fig. 2E), whereas the other two species retain the plesiomorphic state of the internal sac (Fig. 2C) and lack a flagellum (Matsumura and Yoshizawa 2012). The 
average flagellum length is $10.4 \mathrm{~mm}$ in $L$. coronata and $0.93 \mathrm{~mm}$ in L. diversa, although the body length differs only slightly (ca. 5 - $5.5 \mathrm{~mm}$ ) (Matsumura and Suzuki 2008).

Adults were collected in the field in Japan during the reproductive season (late June 2009 and early July 2010). They were kept with their food plants (Commelina communis [Commelinaceae] for $L$. coronata and L. diversa; Lycium chinense [Solanaceae] for L. decempunctata; Oryza sativa [Poaceae] for O. oryzae; summarized in Kimoto and Takizawa 1994) in plastic bags which were placed in an incubator $\left(16 \mathrm{~L}, 8 \mathrm{D}, 27 \pm 1{ }^{\circ} \mathrm{C}\right)$. We collected eggs from the adults and reared them in plastic cups $(13.5 \times 8.5 \times 3.3 \mathrm{~cm})$ with the food plants. After they formed cocoons, we separated individuals every day and kept them in the same condition as the adults.

Criocerine species usually produce the pupal cocoon at the fourth (=final) larval stage (Schmitt 1988; Jolivet and Hawkeswood 1995) and stop consuming food before pupation. Since the differentiation of imaginal discs in holometabolan insects starts after they stop feeding (reviewed in Emlen and Nijhout 2000), we used individuals after the formation of the cocoon for observations. As the life cycles of the four species are quite similar under the same environmental condition (ca. two days in the prepupal stage and ca. five days in the pupal stage [four days only in $O$. oryzae]; YM pers. obs.), we fixed the individuals of each species at regular intervals (i.e. every day) for histological examination. Habitus of the prepupa and pupae of $L$. $(L$.) coronata were visualized in Fig. 3.

\section{(b) Histological techniques}

Larvae, pupae, and adults collected from the rearing stocks were fixed in FAE solution (ethanol: formalin: acetic acid $=16: 6: 1$ ) after perforating the integument with a fine pin, and then stored in $70 \%$ ethanol. We used methacrylate resin Technovit 7100 (Kulzer, Germany) as embedding medium, following the method described by Machida et al. (1994). The abdomen was removed from the remaining body for better penetration. It was then dehydrated in a graded alcohol series, immersed in acetone, and then infiltrated with the resin. In some cases, we immersed the abdomen in hexane (after the acetone) to remove cuticular hydrocarbons to facilitate the medium infiltration. The final step of infiltration was performed in an automatic vacuum infiltrator for one night. Methacrylate-embedded specimens were sectioned at 4-5 $\mu \mathrm{m}$ thickness using a semi-thin microtome (H-1500, BioRad) equipped with a tungsten carbide steel knife (Superhard Knife, Meiwa). Sections were stained with $1 \%$ Delafield's haematoxylin for one night and $0.5 \%$ eosin G for one hour. We observed the slides using a light microscope (Zeiss Axiophot, Germany) and 
took photos with a digital camera (Coolpix 990, Nikon). Based on the photos, we reconstructed three dimensional images using graphic software Mercury Amira ${ }^{\circledR} 4.1 .2$ (Template Graphics Software, USA) and Autodesk Maya ${ }^{\circledR} 7.0$ (Autodesk, USA).

\section{(c) Terminology and orientation of figure presentation}

We mainly followed the classical terminology for the male coleopteran intromittent organ (Sharp and Muir 1912; Lindroth 1957; Snodgrass 1957; Lindroth and Palmén 1970). A modified terminology was used in some works on the genitalia of the phytophagan cucujiform subgroups (Verma 1969: morphogenesis of the intromittent organ of a chrysomelid beetle; Wanat 2007: comparative morphology of male genitalia with a broad taxon sampling from Curculionoidea and Chrysomeloidea). However, as the "classic" terminology was mainly adopted in recent entomology textbooks (e.g. Lawrence and Britton 1991; Lawrence et al. 2010), we used this system consistently to minimize nomenclatorial confusion.

In Coleoptera, a torsion of the aedeagus (i.e. the irreversible rotation along the longitudinal axis during morphogenesis) is known (e.g. Verma 1969, 1971, 1972, 1994; Lindroth and Palmén 1970; briefly reviewed by Hünefeld et al. 2011) in different groups. We could observe a morphogenetic process resulting in a dorso-ventrally reversed position of the whole intromittent organ of Criocerinae during the pupal stage. Torsion probably occurs in the species we used, but we still have to investigate the tracheal and nervous systems to confirm this. Therefore, as a temporal action, we describe a dorso-ventral axis on the assumption that they have a position typical for insects as shown in Fig. 2B.

\section{Results}

Qualitative differences were observed in the morphogenetic process between the species with and without the flagellum. In contrast within the two types, i.e. with and without flagellum, respectively, it was relatively uniform. The morphogenesis is outlined in the following focusing on the differences between the species with and without flagellum.

\section{(a) The morphogenesis of the skeleton of the aedeagus (Figs. 4, 5)}

The differentiation of the imaginal disc of the aedeagus starts in the individuals within 24 hours after the formation of the cocoon. Before pupation, the weakly differentiated imaginal disc is broad and flat (Fig. 4 A, B), but after pupation it starts elongating accompanying the ingrowth of 
the ejaculatory duct, and becomes more slender (Fig. 4). At the early pupal stage a second connecting membrane (SCM), a very short ejaculatory duct (ED) and vasa defferentia (VD), and an aedeagus (A) are already recognizable (Fig. 4C, D, J, K). In the 2nd day of pupation a basal piece of the tegmen (T) appears (Fig. 4E, L, M). Subsequently a border between the internal sac (IS) and median lobe (ML) becomes recognizable in the 2nd (Fig. 4E) or 3rd (Fig. 4G, O, highlighted by arrowheads) day pupae. This means that the internal sac, which is usually stored in the median lobe in the adults stage, is initiated by a constriction at the posterior area of the presumptive aedeagus. After 4 days in pupae of $O$. oryzae and 4 or 5 days in pupae of the other species the internal sac is placed within the wall of the median lobe (this condition is the same as in the adult stage) (Fig. 4I, P, Q).

Only in the species with a flagellum the posterior end of the internal sac starts to invaginate (Fig. 4L, M, see also Fig. 4F) on the 2nd day in L. (L.) coronata (Fig. 5 C) and on 2nd or 3rd day in L. (L.) diversa (Fig. 5B), and the area grows to form the pocket (P) storing the flagellum. Subsequently the invaginated wall extends longitudinally within the tube of the median lobe and internal sac and occupies the posterior three-fourths of it in the 3rd day pupae of L. (L.) coronata (Fig. 4N, O, white broken line). The invagination starts from the dorsal side of the internal-sac sclerite (ISS), which causes a rotation of the sclerites and the opening of the ejaculatory duct during the development of the pocket (Fig. 4L, N, P), whereas the membrane dorsal to ISS does not invaginate in L. (M.) decempunctata and O. oryzae. Internal-sac sclerites differentiate from thickened areas of the epidermis (Fig. 5B, C) and their secretion, i.e. the cuticle. The basal part of the flagellum is formed as a protrusion of the epidermal layer (Fig. 5C, D). In contrast its proximal part consists only of cuticle, lacking an epidermal layer in the available histological sections: this is shown in the two sections of the flagellum in Fig. 5E, with the dorsal one lacking an epidermal layer.

\section{(b) The morphogenesis within the median lobe (Figs. 6, 7)}

Isolated cells were observed in the lumen of the aedeagus in the prepupal stage (Fig. 6A). Most of them differentiate as muscles in latter stages (Fig. 6B, C). At the 2nd day of pupation, the differentiating muscles are inserted already on the inner surface of the pocket for the flagellum (Fig. 6C, D) and around the median foramen (an anterior opening of the median lobe). Even after the insertion on the pocket is established, the surface of it continues to expand during the 3rd day following the growth of the median lobe. The striation of the muscles becomes visible from the 
4th day of pupation and becomes distinct on the 5th day (final day of pupae) (Fig. 6E, F, G).

Conspicuous differences were visible in the transverse planes of the intromittent organ after the 2nd day of pupation between the species with and without flagellum (Fig. 7). Only thin muscle bundles are present in the latter, which also display very wide open spaces in the median lobe + internal sac (Fig. 7A, B, C, D). In contrast, the inner space of the median lobe + internal sac is crowded with the invaginated epidermal layer in the species with the flagellum (Fig. 7E, F, $\mathrm{G}, \mathrm{H})$.

\section{Discussion}

\section{What were novelties for this group?}

In the present study, we observed that the conspicuous invagination of the posterior end of the internal sac occurs only in the species with the flagellum, i.e. Lema (Lema) coronata and L. (L.) diversa (Figs. 4, 5). The invaginated area differentiates into the pocket of the flagellum (Fig. 4L, $\mathrm{N}, \mathrm{P}$ ), and the invagination causes a rotation of the involved sclerites, which in turn causes a $360^{\circ}$ rotation of the tip of the ejaculatory duct (Fig. 4L, L, N). The rotation of the sclerites was considered as the key novelty for the acquisition of the pocket and flagellum (Matsumura and Yoshizawa 2012), which implies that the invagination is a key factor for causing the qualitative differences in the internal sac structure between the species with and without flagellum.

From the morphological point of view, the flagellum and the specialized pocket of species of Neolema (Fig. 2D) and Lilioceris (Fig. 2F) is also mainly formed by a rotation of the entire sclerites, as it is the case in the subgenus Lema (Matsumura and Yoshizawa 2012). Therefore, the similar conditions in adults of Neolema sp. and Lilioceris sp. probably originated by a mechanism identical or very similar to that observed in the subgenus Lema, i.e. the specialized pocket is formed by the invagination of the end of the internal sac and a resultant rotation of sclerites. Although a phylogenetic hypothesis covering all criocerine genera and subgenera is not available yet, morphological observations suggest that Lilioceris is not closely related with Lema and Neolema (Seeno and Wilcox 1982; Schmitt 1985a, b, 1988; Fig. 2A). Schmitt (1985a, b) suggested that the Lilioceris lineage separated in an early stage of the splitting events in the subfamily Criocerinae. This suggests that the common developmental background of the formation of the specialized pocket has already been acquired by the last common ancestor of Criocerinae or even earlier.

The invagination of the posterior end of the internal sac has never been reported in the 
morphogenesis of the aedeagus of beetles without flagellum and pocket (Muir 1918; Rruthi 1924; Metcalfe 1932; Pradhan 1949; Srivastava 1953; Verma 1969). This indicates that this is the newly added feature in Criocerinae, i.e. an evolutionary novelty. The process of invagination itself is of course not novel. Invagination of a cell layer is a common process in the ontogeny of organisms (e.g. gastrulation), and a less conspicuous invagination was also observed when the median lobe and internal sac are differentiated (Fig. 4G, O, arrow head), and also during the formation of the median lobe and parameres of other groups of beetles (Muir 1918; Rruthi 1924; Metcalfe 1932; Pradhan 1949; Srivastava 1953; Verma 1969). Therefore it is very likely that the invagination from the posterior end of the internal sac was caused by a co-option of pre-existing genetic systems, which produced the specialized state of the internal sac in the criocerine species with the flagellum.

As pointed out by Matsumura and Yoshizawa (2012), the musculature is also affected by the acquisition of novelties (compare attachment places shown in Fig. $2 \mathrm{C}$ and E). As there is no equivalent of the pocket in the species without the flagellum, the insertion of muscles on its surface (Fig. 6C, D) is also a novel feature for the species which acquired this structure. The muscles have to be inserted on specific areas to make the pocket functional, as its membrane is withdrawn by their contractions, which causes the withdrawal of the flagellum at the end of copulation (Matsumura and Yoshizawa 2010). Otherwise, the specialized and integrated structure would have been eliminated by natural selection due to its maladaptive character. The position of muscle insertions is determined by the existence and position of muscle pioneers derived from mesodermal cells, which form scaffolds for muscle attachments (e.g. Ho et al. 1983; Rivlin et al., 2000). This implies that just placing the pocket in the passway of developing muscles is not sufficient for the appropriate placement of their insertions. It is likely that changes of the arrangement of muscle insertion's morphogens co-occured with the formation of the specialized pocket of the flagellum. This suggests that the formation of the pocket and modified muscle arrangements have evolved simultaneously in species of the subgenus Lema and the genera Neolema and Lilioceris. To resolve the mechanisms of the coevolution of plural novelties, it is necessary to uncover the genetic mechanisms underlying the morphogenetic process.

\section{Evolutionary mechanisms of correlated growth of the flagellum and pocket}

Our observations show that the pocket is formed by the invagination and rapid growth of the invaginated wall in $L$. (L.) coronata and $L .(L$.$) diversa (Figs. 4,5$ ), and that the flagellum is 
formed by a protrusion of the epidermal layer (Fig. 5C, D) and its cuticular secretion (Fig. 5E). As the formations of the pocket and flagellum are apparently different morphogenetic processes, it appears difficult to explain how the flagellum length fits with the pocket size. Nevertheless, the flagellum is perfectly adapted to the folding of the pocket in the reproductive season (this means the flagellum length is consistent with the length of the folds), which enables the beetles to insert and withdraw the long flagellum during copulation (Matsumura and Yoshizawa 2010).

A condition-dependent mechanism through increased cellular sensitivity to signaling through the insulin/insulin-like growth factor (IGF) (proposed by Emlen et al. 2012) may play a role in the correlated growth of the flagellum and pocket size. Emlen et al. (2012) showed that the extreme growth of the sexually selected horn of the rhinoceros beetle is controlled by increased cellular sensitivity to signaling through IGF, and that this was also related to nutrition conditions (Shingleton et al. 2007, see also supplemental materials in Emlen et al. 2012). In constrast, in the same species, the entire intromittent organ is not sensitive to the nutrition condition and the insulin/IGF passway perturbation (Emlen et al. 2012). However, even in the formation of insect genitalia (including intromittent organs) evolutionary trajectories were assumed to be variable (Tatsuta et al. 2007), and it is possible that only the flagellum and pocket size reflect the condition of the individuals. Actually the length of the flagellum varies more strongly than the body length, even though the length of the median lobe is less variable (YM unpublished data). This model would make it easier to explain the correlated growth of size between the flagellum and pocket. If it really applies to the criocerine intromittent organ, it would be apparently controlled by a widely adopted regulatory system for size control. This would reduce the impediment of acquiring a functional extremely elongated flagellum.

\section{Why was a parallel evolution of plural novelties possible?}

Our observations show that a very large open space is enclosed by the aedeagus throughout the ontogeny in the species retaining the plesiomorphic state (Fig. 7 E, F, G, H, see also Fig. 2C). This area is largely occupied by the pocket in the species with the flagellum (Fig. 7 A, B, C, D). This means that no physical obstacle is present for the pocket formation in the aedeagus throughout the ontogenetic process. The open space enables the intromittent organ to accommodate new elements.

In addition, the time lag between the differentiation and insertion of muscles also facilitates the parallel acquisition of the functional and extremely elongated flagellum. Muscles must be 
arranged adequately on the substratum or ground and attain a suitable length to function adequately. We found that the insertion of muscles occurred on the presumptive pocket in the 2 nd day of pupation (Fig. 6C, D). However, the differentiation of muscles is not completed until the 4th or 5th day of pupation (last day of pupae) when the pocket formation is also completed (Fig. $6 \mathrm{E}, \mathrm{G})$. This suggests that the muscle length is a comparatively adjustable feature in the context of evolution, at least in this group and in this structural and developmental context. Mayr (1960) and Kirschner and Gerhart (2005) strongly emphasized that such flexibilities in morphogenetic processes must play a crucial rule in acquisition of novelties. However, the necessity of simultaneous origins of plural novelties potentially impedes their evolution. As discussed above, this can be overcome by the co-option of previously acquired regulatory systems in morphogenesis. In the most parsimonious scenario the involved mutations are accumulated and integrated as cryptic genetic variation or acquired as genetic accommodation as discussed in the context of the evolution of novelties or innovations (e.g. Mary 1960; Kirschner and Gerhart 2005; Brakefield 2006, 2011; Moczek 2007, 2008; Shubin et al. 2009; Müller 2010; Scotland 2010; Blount et al. 2012).

Our study shows that comparatively simple modifications in morphogenesis can cause apparently large differences observed in the adult stage, and that new elements can be added in the absence of physical obstacles. These facts reduce impediments for criocerine beetles to acquire novelties several times. In future investigations a comparative study of genetic mechanisms involved in criocerines with and without the flagellum should have high priority. This will likely help to reveal mechanisms of the synchronous acquisition of plural interconnected novelties.

\section{Acknowledgments}

We express our thanks to T Yamamoto, Y Ishibashi, K Shimada, and K Toga for collecting the larvae and adults of Lema (Microlema) decempunctata used in this study, and also to A Iwasaki, N Miyake, K Onodera, who kindly helped us to collect the adults of Oulema oryzae. We really appreciate technical support provided by Y Uchifune-Jintsu, M Fukui, K Ishitani, K Sekiya, Y Mashimo, K Maekawa, S Niitsu, and F Hünefeld, and T Saigusa for valuable advice. This study was supported by Research Fellowships of the Japan Society for the Promotion of Science (JSPS) for Young Scientists and Studying abroad to YM and Grant-in-Aid from the JSPS (Scientific Research C: 21570089) to RM. 


\section{References}

Atallah, J., Watabe, H., and Kopp, A. 2012. Many ways to make a novel structure: a new mode of sex comb development in Drosophila. Evol. Dev. 14: 476-483.

Blount, Z. D., Barrick, J. E., Davidson, C. J., and Lenski, R. E. 2012. Genomic analysis of a key innovation in an experimental Escherichia coli population. Nature 489: 513-518.

Brakefield, P. M. 2006. Evo-devo and constraints on selection. Trends Ecol. Evol. 21: 362-368.

Brakefield, P. M. 2011. Evo-devo and accounting for Darwin's endless forms. Philos. Trans. R. Soc. B Biol. Sci. 366: 2069-2075.

Brylski, P., and Hall, B. K. 1988. Ontogeny of a macroevolutionary phenotype: the external cheek pouches of geomyoid rodents. Evolution 42: 391-395.

Dugon, M. M., Hayden, L., Black, A., and Arthur, W. 2012. Development of the venom ducts in the centipede Scolopendra: an example of recapitulation. Evol. Dev. 14: 515-521.

Emlen, D. J., and Nijhout, H. F. 2000. The development and evolution of exaggerated morphologies in insects. Annu. Rev. Entomol. 45: 661-708.

Emlen, D. J., Warren, I. A., Johns, A., Dworkin, I., and Lavine, L. C. 2012. A Mechanism of Extreme Growth and Reliable Signaling in Sexually Selected Ornaments and Weapons. Science 337: 860-864.

Hendrickson, H., and Rainey, P. B. 2012. How the unicorn got its horn. Nature 489: 504-505.

Ho, R. K., Ball, E. E., and Goodman, C. S. 1983. Muscle pioneers: large mesodermal cells that erect a scaffold for developing muscles and motonuerones in grasshopper embryos. Nature 301: 66-69.

Hünefeld, F., Marvaldi, A. E., Müller, B., Lawrence, J. F., and Beutel, R.G. 2011. The male postabdomen of the "ancestral" archostematan beetle Tetraphalerus bruchi Heller, 1913 (Ommatidae) and its phylogenetic significance. Arthropod Struct. Dev. 40: 146-158.

Jolivet, P., and Hawkeswood, T. J. 1995. Host-plants of Chrysomelidae of the world. Backhuys Publishers, Leiden.

Kaji, T. 2010. Ontogeny and function of the fifth limb in Cypridocopain Ostracods. Zool. Sci. 27: 673-677.

Kaji, T. 2012. Developmental process of musculoskeletal integration in ostracod antenna. Arthropod Struct. Dev. 41: 177-185.

Kaji, T., Maran, B. A. V., Kondoh, Y., Ohtsuka, S., Boxshall, G. A., and Tsukagoshi, A. 2012. 
The lunule of caligid copepods: an evolutionarily novel structure. Evol. Dev. 14: 465-475.

Kaji, T., Møller, O. S., and Tsukagoshi, A. 2011. A bridge between original and novel states: ontogeny and function of "suction discs" in the Branchiura (Crustacea). Evol. Dev. 13: 119126.

Kaji, T., and Tsukagoshi, A. 2008. Origin of the novel chemoreceptor Aesthetasc "Y" in Ostracoda: morphogenetical thresholds and evolutionary innovation. Evol. Dev. 10: 228-240.

Kaji, T., and Tsukagoshi, A. 2010. Heterochrony and modularity in the degeneration of mazillopodan nauplius eyes. Biol. J. Linn. Soc. 99: 521-529.

Kimoto, S., and Takizawa, H. 1994. Leaf beetles (Chrysomelidae) of Japan. Tokai University Press, Tokyo. (In Japanese with English keys and brief synonym list.)

Kirschner, M. W., and Gerhart, J. C. 2005. The Plausibility of Life, Resolving Darwin 's Dilemma. Yale University Press, New Haven.

Lawrence, J. F., Beutel, R. G., Leschen, R. A. B., and Ślipiński, S. A. 2010. Chapter 2. Glossary of Morphological Terms. In R. A. B. Leschen, R. G. Beutel and J. F. Lawrence (eds.). Handbook of Zoology, Coleoptera Volume 2: Morphology and Systematics (Elateroidea, Bostrichformia, Cucujiformia partim). Walter de Gruyter, Berlin. pp. 9-20.

Lawrence, J. F., and Britton, E. B. 1991. Coleoptera (beetles). In CSIRO, Division of entomology (ed.). The Insect of Australia, Second edition, vol. 2. Cornell University Press, Ithaca, N.Y. pp. 543-683.

Lindroth, C. H. 1957. The principal terms used for male and female genitalia in Coleoptera. Opusc. Entomol. 22: 241-256.

Lindroth, C. H., and Palmén, E. 1970. Coleoptera. In S. L. Tuxen (ed). Taxonomist's Glossary of Genitalia in Insects, Second revised and enlarged edition. Munksgaard, Copenhagen. pp. 8088.

Machida, R., Nagashima, T., and Ando, H. 1994. Embryonic development of the jumping bristletail Pedetontus unimaculatus Machida, with special reference to embryonic membranes (Hexapoda: Microcoryphia, Machilidae). J. Morphol. 220: 147-165.

Matsumura, Y., and Akimoto, S. 2009. Mating behavior and genital damage during copulation in the leaf beetle Lema coronata (Chrysomelidae: Criocerinae). Entomol. Sci. 12: 215-217.

Matsumura, Y., and Suzuki, K. 2008. Comparative morphology of internal reproductive systems in leaf beetles of the Donaciinae and Criocerinae (Coleoptera: Chrysomelidae) and its implication for the phylogeny. Zootaxa 1845: 1-32. 
Matsumura, Y., and Yoshizawa, K. 2010. Insertion and withdrawal of extremely elongated genitalia: a simple mechanism with a highly modified morphology in the leaf beetle, Lema coronata. Biol. J. Linn. Soc. 99: 512-520.

Matsumura, Y., and Yoshizawa, K. 2012. Homology of the internal sac components in the leaf beetle subfamily Criocerinae and evolutionary novelties related to the extremely elongated flagellum. J. Morphol. 273: 507-518.

Mayr, E. 1960. The emergence of evolutionary novelties. In S. Tax (ed.). Evolution after Darwin. University of Chicago Press, Chicago. pp. 349-380.

Metcalfe, M. E. 1932. The Structure and Development of the Reproductive System in the Coleoptera with notes on its Homologies. Q. J. Microsc. Sci. 75: 49-129.

Moczek, A. P. 2007. Developmental capacitance, genetic accommodation, and adaptive evolution. Evol. Dev. 9: 299-305.

Moczek, A. P. 2008. On the origins of novelty in development and evolution. BioEssays 30: 432447.

Muir, F. 1918. XII. Notes on the ontogeny and morphology of the male genital tube in Coleoptera. Trans. Entomol. Soc. Lond. 1918: 223-229.

Müller, G. B. 2010. Epigenetic Innovation. In M. Pigliucci and G. B. Müller (eds.). Evolution the Extended Synthesis. MIT press, Cambridge. pp. 307-333.

Müller, G. B., and Wagner, G. P. 1991. Novelty in evolution: restructuring the concept. Annu. Rev. Ecol. Syst. 22: 229-256.

Müller, G. B., and Wagner, G. P. 2003. Innovation. In B. K. Hall and W. M. Olson (eds.). Keywords and Concepts in Evolutionary Developmental Biology. Harvard University Press, Cambridge. pp. 218-227.

Nagashima, H., Sugahara, F., Takechi, M., Ericsson, R., Kawashima-Ohya, Y., Narita, Y., and Kuratani, S. 2009. Evolution of the turtle body plan by the folding and creation of new muscle connections. Science 325: 193-196.

Neufeld, C. J., and Palmer, A. R. 2008. Precisely proportioned: intertidal barnacles alter penis form to suit coastal wave action. Proc. R. Soc. Lond. B Biol. Sci. 275: 1081-1087.

Page, L. R. 2000. Development and evolution of adult feeding structures in Caenogastropods: overcoming larval functional constraints. Evol. Dev. 2: 25-34.

Pradhan, K. S. 1949. On the structure and post-embryonic development of the male genital organs of the woolly bear, Anthrenus fasciatus Herbst (Coleoptera, Dermestidae). J. Zool. Soc. 
India 1: 49-56.

Prum, R. O. 1999. Development and evolutionary origin of feathers. J. Exp. Zool. B Mol. Dev. Evol. 285: 291-306.

Rivlin, P. K., Schneiderman, A. M., and Booker, R. 2000. Imaginal Pioneers Prefigure the Formation of Adult Thoracic Muscles in Drosophila melanogaster. Dev. Biol. 222: 450-459.

Rruthi, H. M. 1924. On the post-embryonic development and Homologies of the male genital organs of Tenebrio molitor L. (Coleoptera). Proc. Gen. Meet. Sci. Business Zool. Soc. Lond. 94: $857-868$.

Roth, G., and Wake, D. B. 1989. Conservatism and Innovation in the Evolution of Feeding in Vertebrates. In D. B. Wake and G. Roth (eds.). Complex Organismal Functions: Integration and Evolution in Vertebrates. John Wiley and Sons, Chichester. pp. 7-21.

Schmitt, M. 1985a. Versuch einer phylogenetisch-systematischen analyse der Criocerinae (Coleoptera, Chrysomelidae). Zool. Beitr. N. F. 29: 35-85.

Schmitt, M. 1985b. On the phylogeny of the Criocerinae (Coleoptera, Chrysomelidae). Entomography 3: 393-401.

Schmitt, M. 1988. 28. The Criocerinae: Biology, Phylogeny and Evolution. In P. Jolivet, E. Petitpierre and T. H. Hsiao (eds.). Biology of Chrysomelidae. Kluwer Academic Publishers, Dordrecht. pp. 475-495.

Scotland, R. W. 2010. Deep homology: A view from systematics. Bioessays 32: 438-449.

Seeno, T. N., and Wilcox, J. A. 1982. Leaf beetle genera (Coleoptera: Chrysomelidae). Entomography 1: 1-221.

Sharp, D., and Muir, F. A. G. 1912. The comparative anatomy of the male genitalia tube in Coleoptera. Trans. Entomol. Soc. Lond. 1912: 477-642.

Shingleton, A. W., Frankin, W. A., Flatt, T., Nijhout, H. F., and Emlen, D. J. 2007. Size and shape: the developmental regulation of static allometry in insects. BioEssays 29: 536-548.

Shubin, N., Tabin, C., and Carroll, S. 2009. Deep homology and the origins of evolutionary novelty. Nature 457: 818-823.

Snodgrass, R. E. 1935. Principles of Insect Morphology. McGraw-Hill Book Co., New York and London.

Snodgrass, R. E. 1957. A revised interpretation of the external reproductive organs of male insects. Smithsonian Misc. Coll. 135 (6): 1-61.

Srivastava, U. S. 1953. On the post-embryonic development of the male genital organs (external 
and internal) of Tribolium castaneum Herbst (Coleoptera Tenebrionidae). Indian J. Entomol. 15: $352-361$.

Tajiri, R., Misaki, K., Yonemura, S., and Hayashi, S. 2010. Dynamic shape changes of ECM-producing cells drive morphogenesis of ball-and-socket joints in the fly leg. Development 137: 2055-2063.

Tatsuta, H., Fujimoto, K., Mizota, K., Reinhardt, K., and Akimoto, S. 2007. Distinctive developmental variability of genital parts in the sexually dimorphic beetle, Prosopocoilus inclinatus (Coleoptera: Lucanidae). Biol. J. Linn. Soc. 90: 573-581.

Verma, K. K. 1969. Functional and developmental anatomy of the reproductive organs in the male of Galerucella birmanica Jac. (Coleoptera, Phytophaga, Chrysomelidae). Ann. Sci. Nat. B 12: 139-234.

Verma, K. K. 1971. 'Retournement' of the aedeagus in Chrysomelidae (Coleoptera, Phytophaga). J. Nat. His. 5: 635-642.

Verma, K. K. 1972. The aedeagus, its musculature, and 'retournement' in Aspidomorpha miliaris F. (Coleoptera, Phytophaga, Chrysomelidae). J. Nat. His. 6: 699-719.

Verma, K. K. 1994. 'Retournement' of the aedeagus in Chrysomelidae (Coleoptera). In P. H. Jolivet, M. L. Cox and E. Petitpierre (eds). Novel Aspects of the Biology of Chrysomelidae. Kluwer Academic Publishers, Dordrecht. pp. 355-362.

Wanat, M. 2007. Alignment and homology of male terminalia in Curculionoidea and other Coleoptera. Invertebr. Syst. 21: 147-171.

Figure legends

Figure 1. Schema and terminology of the criocerine the male intromittent organ (aedeagus) in copula. The internal sac and flagellum are stored in the median lobe in repose. ED: ejaculatory duct; F: flagellum, FCM: first connecting membrane; IS: internal sac; ML: median lobe; PG: primary gonopore, SCM: second connecting membrane; T: tegmen.

Figure 2. Schematics of the intromittent organ's movement and transformation history in Criocerinae. All figures of the intromittent organs were drawn in lateral view, and the right side is corresponding to posterior end of the male body. A: a phylogenetic hypothesis of Criocerinae (after Schmitt 1985a, b), our preliminary analysis based on molecular data shows that Neolema is distantly related from Lema (Lema); a thickened line and black areas indicate that some of the members acquired a pocket in their intromittent organ. B: intromittent organ's movement, only abdomens are shown. C, D, E, F: internal sac structure; C was presumed to be the plesiomorphic state and be observed widely in Criocerinae, and D, E, F was derived from $\mathrm{C}$; although the appearance of $\mathrm{D}, \mathrm{E}, \mathrm{F}$ is completely different among them, a transformation pattern can be explained by rotation of the whole sclerites along the craniocaudal axis. Muscular attachments of Neolema sp. and Lilioceris sp. are not known. 
ED: ejaculatory duct; F: flagellum; IS: internal sac; ISS: internal-sac sclerites; MA: muscle attachments; ML: median lobe; P: pocket.

Figure 3. Habitus of prepupa and pupae in L. (L.) coronata. A: Prepupa 2nd day. B: Pupa 1st day. C: Pupa 5th day. Scale bars are $1 \mathrm{~mm}$.

Figure 4. Outlines and comparison of the morphogenetic processes of the intromittent organ during prepupal (A, B) and pupal (C-Q) stages of $L$. (M.) decempunctata (A-I) and L. (L.) coronata (A, B, J-Q). All the figures exhibited as the direction shown in the upper right corner. $\mathrm{A}, \mathrm{C}, \mathrm{G}$, and $\mathrm{K}$ : whole aedeagus in ventral view (the second connecting membrane = $\mathrm{SCM}$ is not shown in $\mathrm{G}$ ); others (B, D, E, F, H, I, J K, L, M, O, P and Q, the second connecting membrane is not shown in $\mathrm{F}$ and $\mathrm{Q}$ ): sagittal plane, right side corresponds to ventral. Arrowheads means the opening of the ejaculatory duct, arrows show a direction of invagination. Ticks in $\mathrm{G}$ indicate contraction between the presumptive median lobe and internal sac. A: aedeagus; ED: ejaculatory duct; F: flagellum; FCM: first connecting membrane; IS: internal sac; ISS: internal-sac sclerites; M: muscles; ML: median lobe; P: pocket for flagellum; SCM: second connecting membrane; T: tegmen; VD: vasa deferentia.

Figure 5. Morphogenesis of the pocket and flagellum. A: schema indicating areas of B, C, D, and E. B, C: almost sagittal sections of posterior part of the presumptive aedeagus, anterior to the top; B is $L$. (L.) diversa, 3rd day pupae; C is $L$. (L.) coronata, 2 nd day pupae. D, E: transverse sections of $L$. $(L$.) coronata, 3rd day pupae, ventral to the top; $\mathrm{D}$ is anterior area of pocket, $\mathrm{E}$ is more posterior to D. A: aedeagus; ED: ejaculatory duct; F: flagellum; ISS: internal-sac sclerites; M: muscles; P: pocket; SCM: second connecting membrane. Scale bars in B, C are $250 \mu \mathrm{m}$ and in D, E are $100 \mu \mathrm{m}$.

Figure 6. Muscles' development of aedeagus in L. (L.) coronata. A: 1st day prepupa, whole presumptive aedeagus shown from coronal plane. B: 2nd day prepupa, whole aedeagus in sagittal view. C: 2nd day pupa, posterior half of aedeagus in almost sagittal view. D: id., posterior area corresponding to the broken line in $\mathrm{C}$ shown from transverse plane. E: 4th day pupa enlarged muscles in transverse view. F: 5th day pupa, whole aedeagus in sagittal view. G: id., enlarged muscles in sagittal view. A: aedeagus; ED: ejaculatory duct; IC: isolated cells; IS: internal sac; M: muscles; P: pocket for flagellum; SCM: second connecting membrane; VD: vasa differentia. Scale bars in A, B, C are $250 \mu \mathrm{m}$, in D is $100 \mu \mathrm{m}$, in F is $500 \mu \mathrm{m}$, and in $\mathrm{E}, \mathrm{G}$ is $50 \mu \mathrm{m}$.

Figure 7. Conspicuous differences observed in morphogenesis between the species with and without the pocket and flagellum. A, B: L. (L.) diversa, 4th day pupa; C, D: L. (L.) coronata, 3rd day pupa; E, F: L. (L.) decempunctata, 3rd day pupa; G, H: O. oryzae, 3rd day pupa. B, C, F, G: histological sections in transverse planes of the middle of the aedeagus. A, D, E, H: schematics of the differences; dotted areas mean inside of the pocket for the flagellum; dark grey areas highlight open spaces. ED: ejaculatory duct; F: flagellum; IS: internal sac; ISS: internal-sac sclerites; M: muscles; ML: median lobe; OP: open space; P: pocket for flagellum; P3: 3rd day after pupation; P4: 4th day after pupation; SCM: second connecting membrane; $\mathrm{T}$ : tegmen. Scale bars are $250 \mu \mathrm{m}$. 


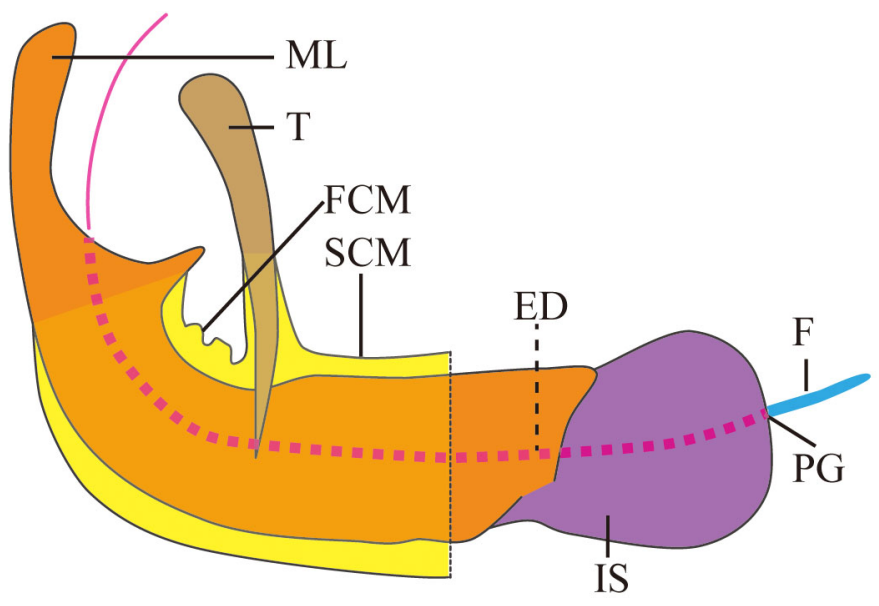



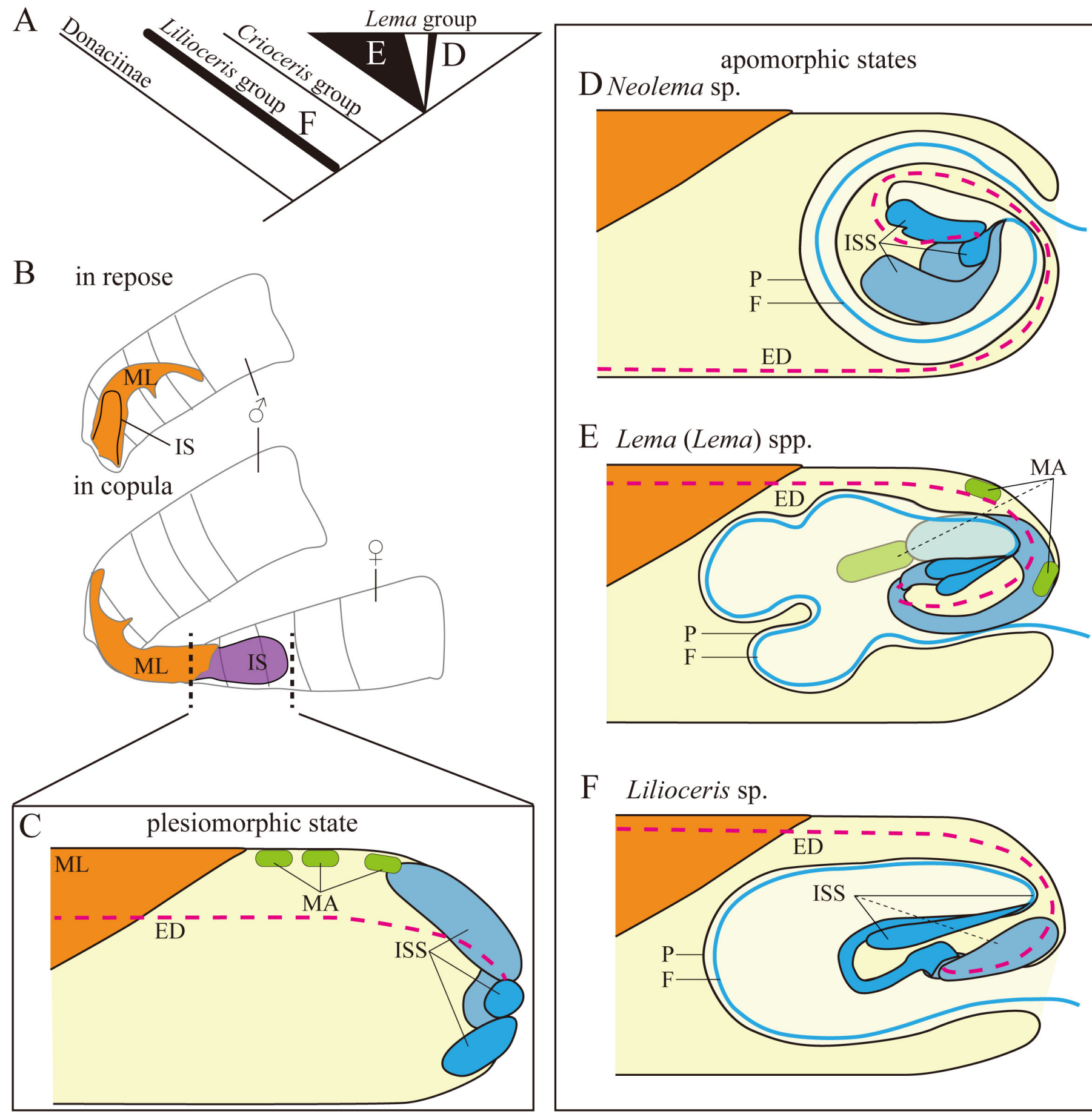

E Lema (Lema) spp.

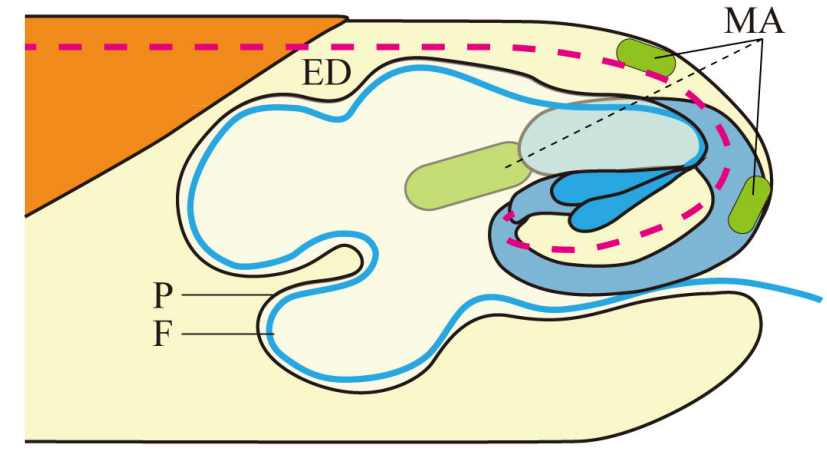

F Lilioceris sp.

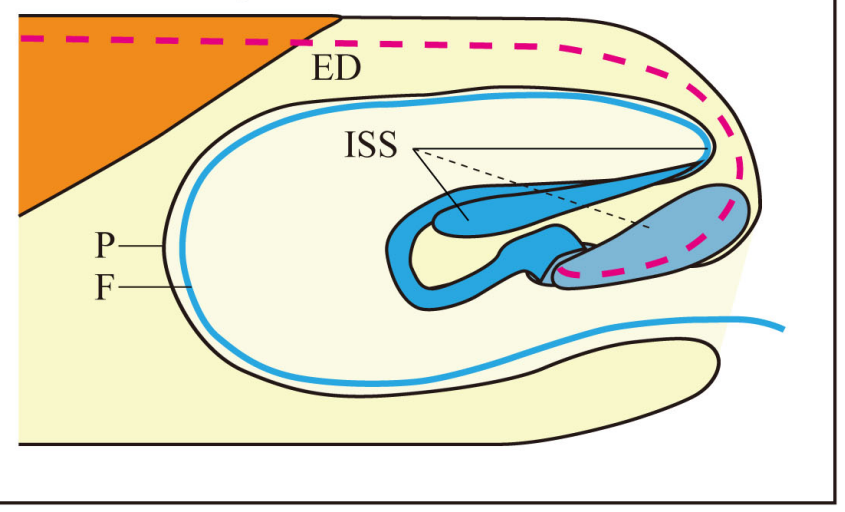




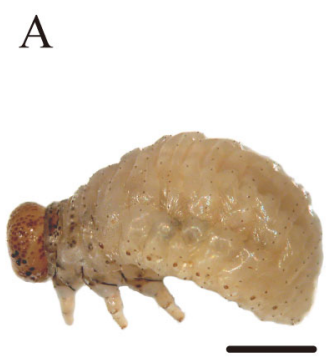

B

C
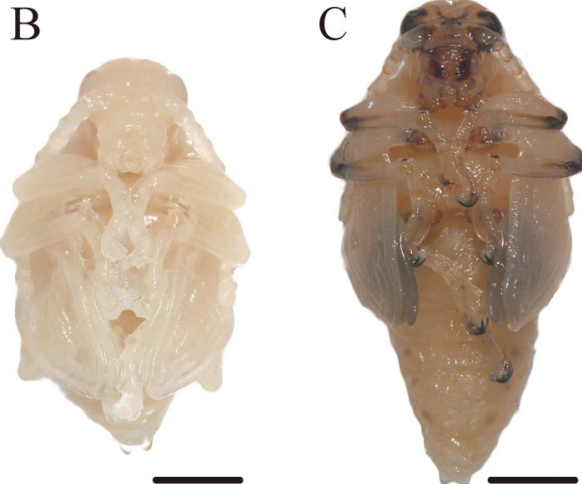


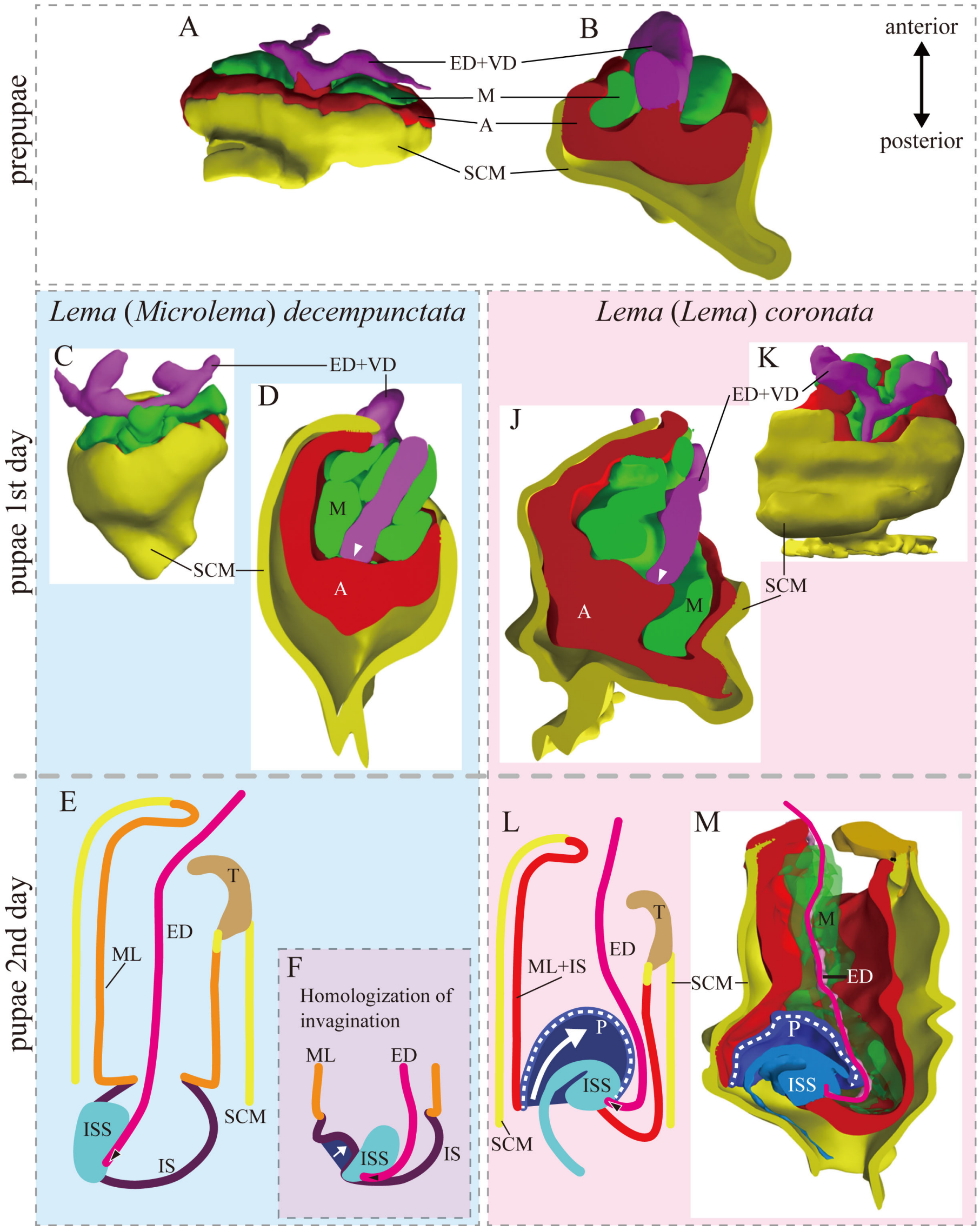




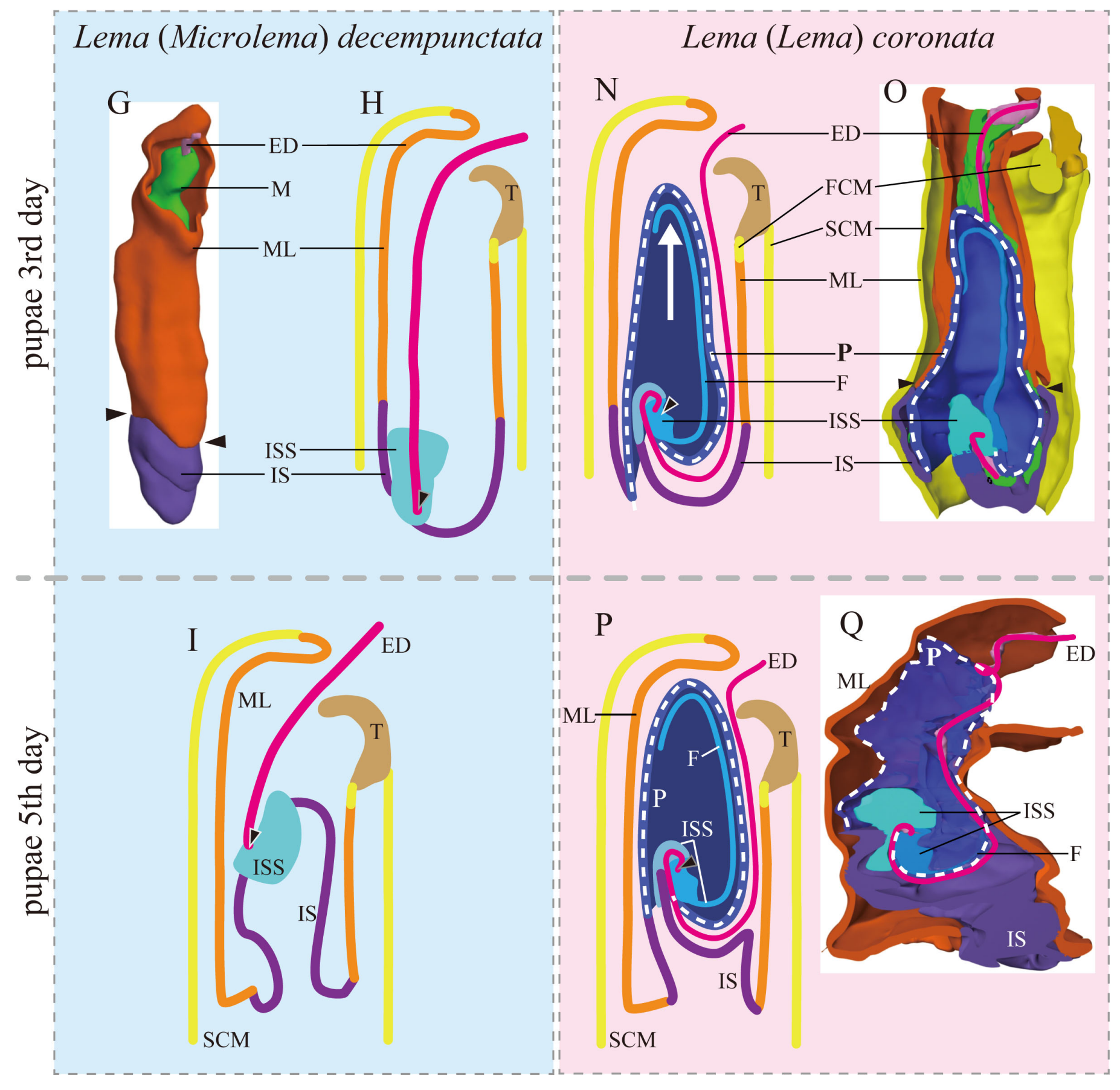



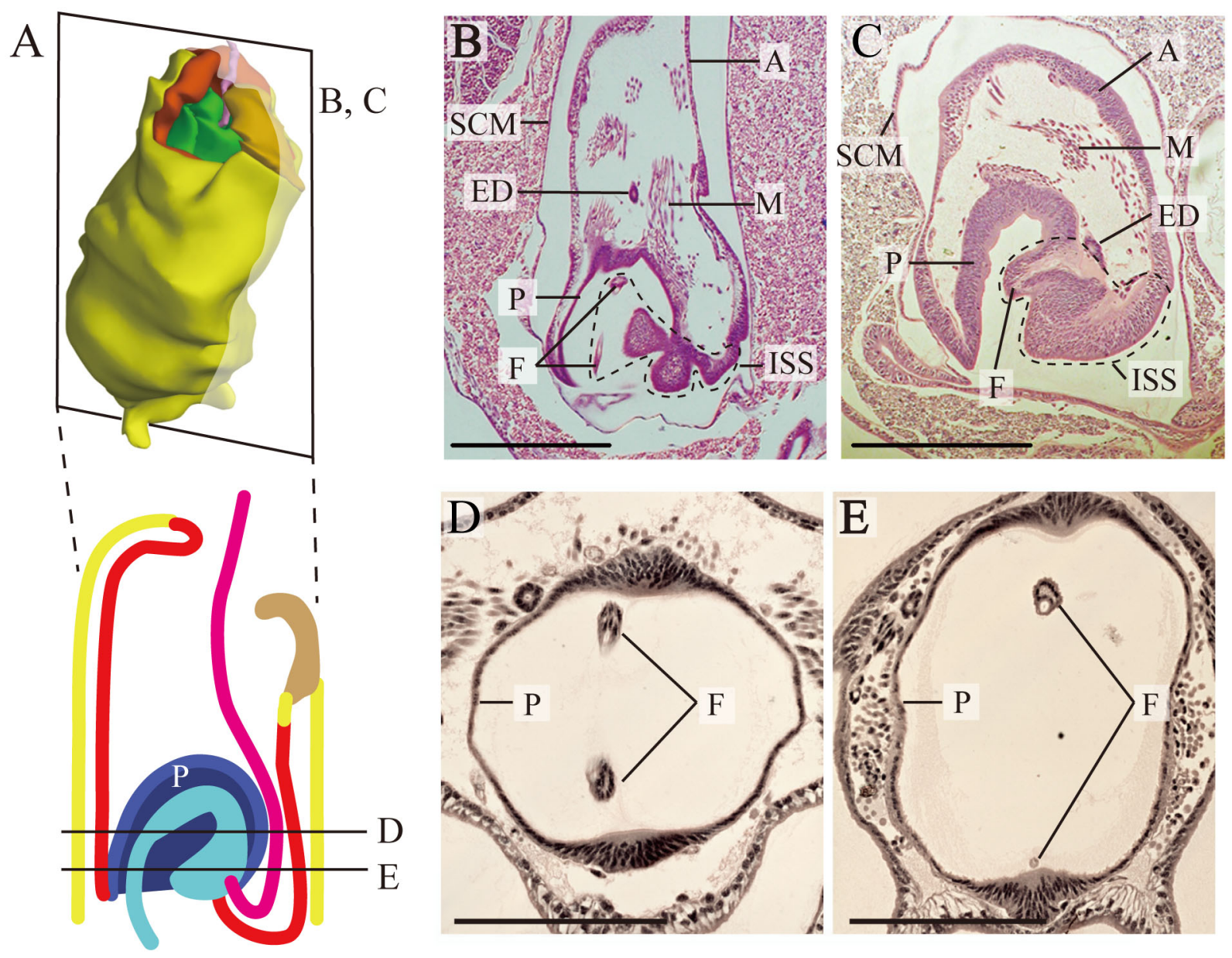

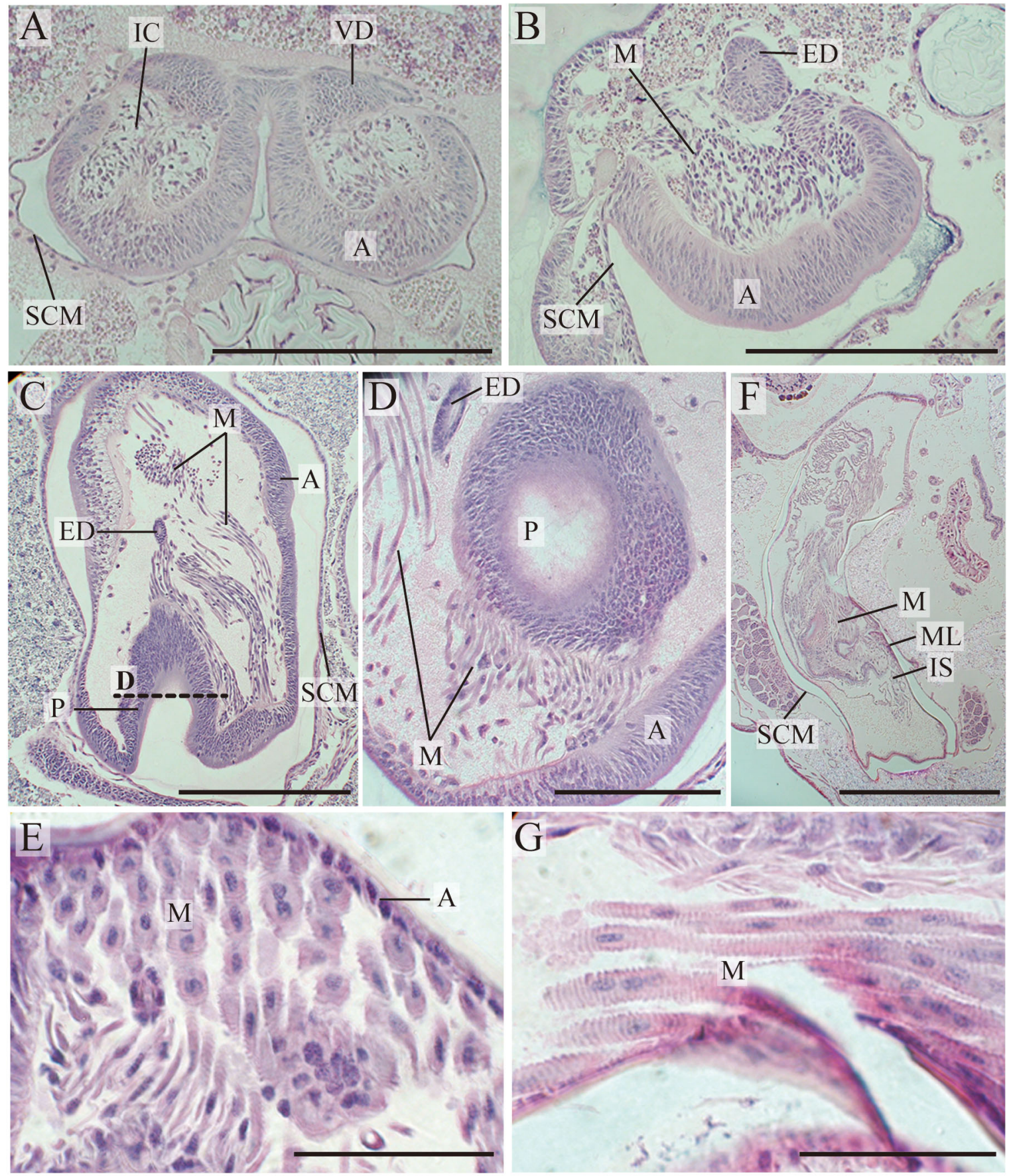

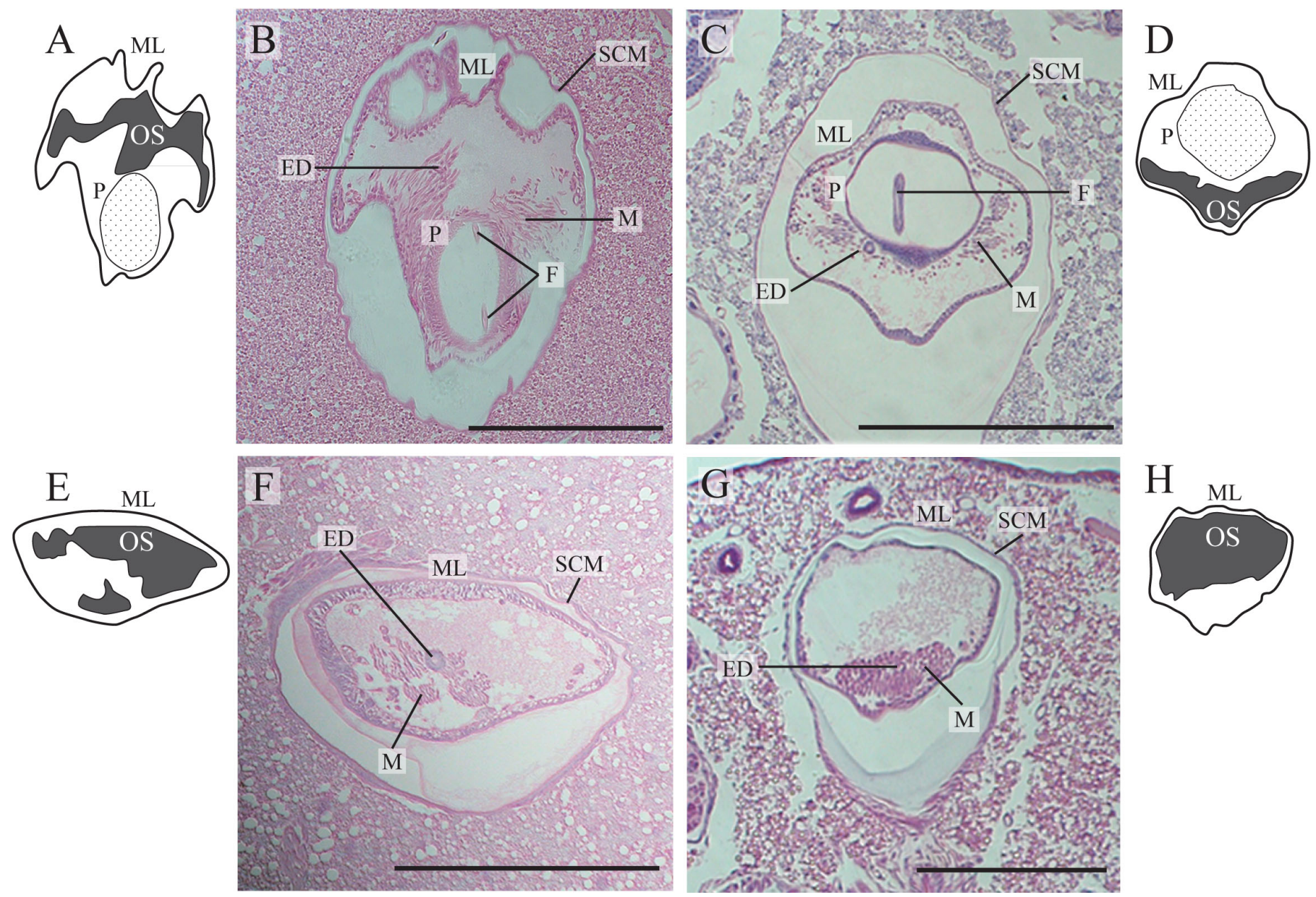